\title{
Modeling Long Probes in Flowing Plasmas using KiPS-2D, a Novel Steady-State Vlasov Solver
}

\author{
Éric Choinière* and Brian E. Gilchrist ${ }^{\dagger}$ \\ University of Michigan, Ann Arbor, Michigan 48109
}

\begin{abstract}
A Consistent steady-state kinetic 2D plasma model and the corresponding computational solver were developed and used for the modeling of long conductive electron-collecting probes in flowing mesosonic plasmas. Sheath asymmetries, not accounted for in previous treatments of ion-collecting probes in flowing plasmas, are modeled here and shown to consititute an important mechanism for the departure from OML theory for electron current collection in the mesosonic regime. The effects of collisions are addressed by dividing the space surrounding the probe into a collisionless computational space and a collisional background plasma. The implementation of the solver consists of successive linearizations of the nonlinear Poisson-Vlasov operator, within a Tikhonov-regularized Newton iterative process. The Finite Element Method is used for the Poisson solver, while the inside-out trajectory tracking procedure is used for the Vlasov solver. The parallel solver allows for the arbitrary velocity distributions of both species within the computational domain, provides an adaptive, unstructured meshing strategy, and allows simulation of very large computational domains. Results show indication of a small enhancement, with respect to OML theory, of the collected current to an electron-attracting probe in a flowing plasma. This enhancement is attributed to the elongation of the pre-sheath into the collisional zone of the plasma, which causes an enhanced density of incoming electrons upstream from the probe, and is seen to dominate the opposing decrease in electron collection due to additional potential barriers created by a wake-side depression of the electric potential. The primary issue is the accumulation of noise in the solution that subsides in spite of the employed Tikhonov regularization, limiting the progress of the iterative scheme. Improvements in the Vlasov solver to reduce the amount of quadrature noise it generates are planned to improve the consistency of solutions.
\end{abstract}

\section{Introduction}

$\mathbf{L}$ ANGMUIR probes and bare space electrodynamic tethers are two important applications involving long electron-collecting conductive cylinders in flowing plasmas. The interpretation of Langmuir probe data in flowing plasma diagnostics, as well as the prediction of current collection to bare space electrodynamic tethers, are lacking an accurate model that accounts for the sheath asymmetries that arise in mesosonic flowing plasmas.

Space electrodynamic tethers offer the opportunity for propellantless propulsion of near-earth orbiting spacecraft, based on the conversion of the geomagnetic force on an electric current along a tether into a propulsive force. ${ }^{1}$ One of the key parameters affecting thrust is the current level flowing

${ }^{*}$ Graduate Student, Radiation Laboratory, EECS Department, AIAA Student Member, echoinie@umich.edu

${ }^{\dagger}$ Professor, Electrical Engineering and Space Sciences, AIAA Senior Member, gilchrst@umich.edu through the tether, which in turn is limited by the amount of electron current collected from the ionosphere. Bare conductive tethers are believed to be very efficient electron collectors when compared to configurations using an insulated tether combined with an end collector. ${ }^{2}$ However, space tethers are moving through the ionosphere at orbital velocities, effectively adding a flow component to the surrounding plasma. It is desirable to assess how the electron collection capability of a cylindrical bare tether, immersed in a flowing plasma, departs from that predicted by the Orbital Motion Limit, which is only valid in the case of thin wires ${ }^{3}$ in non-flowing plasmas.

In both space electrodynamic tether applications and plasma diagnostic devices, a detailed model of the plasma kinetics is required in order to accurately predict the current collection to cylinders of various cross-section geometries in flowing plasmas and/or to extract estimates of the plasma parameters from measured voltage-current characteristic curves. 


\section{Existing Models}

Exact analytical solutions to the symmetric problem of an attracting round conductive cylinder immersed in a non-flowing plasma only exist in the thin sheath (infinite cylinder radius) and orbital-motion (vanishing cylinder radius) limits. The thin-sheath and OML normalized currents are given by ${ }^{4}$

$$
\begin{aligned}
\frac{I_{t s}}{I_{t h}} & =1, \phi>0 \\
\frac{I_{o m l}}{I_{t h}} & =\left\{\frac{2}{\sqrt{\pi}} \sqrt{\phi}+\exp (\phi) \operatorname{erfc}(\sqrt{\phi})\right\}, \phi>0
\end{aligned}
$$

where the thermal current, which normalizes both equations, is given by $I_{t h}=A_{p} n q \sqrt{\frac{e T}{2 \pi m}}$, where $A_{p}$ is the cylinder area, $n$ is the number density of attracted particles in $\mathrm{m}^{-3}, m$ and $q$ are the particle mass and charge, $T$ is the temperature of the species in units of $e V$, and $e=1.6 \times 10^{19} \mathrm{~J} / \mathrm{eV}$. The normalized bias potential $\phi$ is related to the physical bias potential $V$ by $\phi=\frac{V-V_{p}}{T}$, where $V_{p}$ is the plasma potential.

To address cases other than those two limits, a consistent solution to the problem of the non-flowing ion-attracting round cylindrical probe was first provided by Bernstein and Rabinovitz, ${ }^{5}$ who developed a Poisson/Vlasov consistent, 1-D model based on approximations of mono-energetic ions and negligible electron current. The computations consisted of numerically integrating an ordinary differential equation of one variable. Laframboise ${ }^{6}$ later developed a consistent iterative numerical scheme that allowed for Maxwellian distributions (far from the probe) for both the attracted and repelled species, and involved the iterative resolution of a nonlinear system of integral equations, with results applicable to all potential values, temperatures, and probe sizes with respect to the Debye length.

Several authors have also addressed, in a firstorder sense, the problem of ion collection by a round cylindrical probe immersed in a flowing plasma, using the crucial assumption of a radially symmetric potential profile, unaffected by any flow effects. Mott-Smith and Langmuir ${ }^{4}$ derived an asymptotic formula valid in the limit of large speed ratios (relative to the ion thermal velocity) for the current characteristic in the large sheath limit (orbital motion limit). Kanal ${ }^{7}$ derived similar expressions valid in the limit of small speed ratios. Hoegy and Wharton $^{8}$ generalized those results by providing expressions valid for all speed ratios, for the limiting cases of thin sheath, large sheath (orbital motion limit) and retarding regimes.

Godard and Laframboise ${ }^{9}$ went further by devel- oping a numerical model that allowed for all probe radiuses to be considered in the flowing case by using the 1-D cylindrically symmetric potential profiles obtained by Laframboise ${ }^{6}$ as the assumed electric potential.

In the case of the mesosonic regime, where the velocity of the flow is much larger than the ion thermal velocity but much smaller than the electron thermal velocity, only ion collection can be addressed by an approximate solution based on an assumed symmetric potential profile. Such an approximation would show virtually no departure from the non-flowing results in the case of electron collection, due to their large thermal velocity as compared to the speed of the mesothermal flow. In the mesothermal regime, the effects of the flow on the collection of the light species - the electrons- are only indirect. That is, these effects only occur due to the asymmetries in the potential profile around the probe that are induced by the heavier ion species.

Even for the ion-attracting case, the assumption of a symmetric profile could prove erroneous at least in cases showing one or more of these two conditions: ${ }^{9}$

- the probe radius is not small with respect to the Debye length, implying a non negligible and likely asymmetric ion space charge distribution near the collector;

- the ratio of flow energy to bias potential is neither very small (a small flow could only cause small asymmetries) nor very large (in which case the bias potential could not significantly affect the flow).

\section{Proposed 2D Steady-State Kinetic Model}

We present the development and software implementation of a consistent, steady-state, electrodynamic plasma model based on kinetic theory. The solver presented here is best classified as a Vlasov code based on a kinetic description of plasmas. ${ }^{10}$ It differs from Particle-In-Cell methods in that the Vlasov equation is solved directly instead of resorting to a set of discrete macro-particles. It is also distinct from Eulerian Vlasov implementations, ${ }^{11}$ owing primarily to the fact that a steadystate "non time-varying" solution is sought directly, without resorting to incremental time stepping. The steady-state solver presented here bares some similarities with previous work addressing other geometries, ${ }^{12-14}$ and may be regarded as a 2-D extension of the 1-D model developed by Laframboise. ${ }^{6}$

The aim of this model is to numerically solve, self-consistently, the Poisson and Vlasov equations in a steady-state over a large computational region 
around an arbitrarily-shaped 2D conductive body in a collisionless, unmagnetized, flowing 2-species plasma. The implementation of the solver, called KiPS-2D (Kinetic Plasma Solver, 2-dimensional) consists of successive linearizations of the nonlinear Poisson-Vlasov operator, within a Tikhonovregularized Newton iterative process. Following are the main features of the proposed model and solver:

- it allows for the representation of the complete, arbitrary velocity distribution of both plasma species at all sampled locations in the computational domain;

- it can model plasma cross flow in all speed regimes with respect to the thermal velocities of the species;

- it provides a dynamic, adaptive, unstructured meshing strategy, allowing for the resolution of sheath asymmetries induced by the plasma flow and the support of conductive objects with arbitrary 2D geometries;

- it includes a Finite-Element based Poisson solver incorporating a background plasma boundary condition based on asymptotic analytical results; ${ }^{5}$

- it allows for the simulation of very large computational domains in order to accommodate the pronounced pre-sheath elongation (several hundred Debye lengths) along the direction of plasma flow. The variable grid size is tailored locally to efficiently accommodate potential variations. Numerical instabilities resulting from large grid sizes are handled using a Tikhonov regularization process;

- its steady-state Vlasov solver features a parallel implementation based on the PVM library, ${ }^{15}$ allowing it to run on either a single host, a parallel architecture, or a scattered network of workstations based on a MPMD (Multiple Processors, Multiple Data) scheme.

\section{Definition of the Problem}

The basic probe geometry under consideration is an arbitrarily-shaped 2-dimensional object, i.e. a cylinder of arbitrary cross-section geometry and infinite length. Probe end effects are neglected in this treatment, which is hardly an approximation in the case of bare space electrodynamic tethers, which are typically several kilometers long.

This 2D conductive object (referred to as the probe) is immersed in a flowing 2-species unmagnetized plasma with the plasma flow directed along the

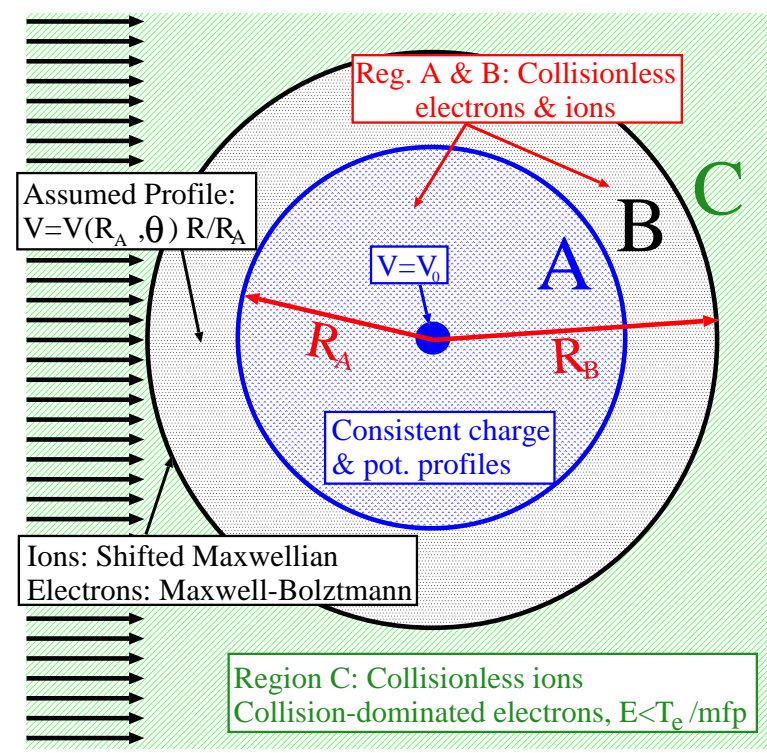

Fig. 1 Geometry of the plasma model. Regions $A$ and $B$ are part of simulation space, while region $C$ defines a boundary condition on the outer edge of region $B$.

$x$ axis. A bias potential $V=V_{0}$ is applied between the probe and a surface located at infinity, $r=\infty$. In order to accurately represent the elongated sheath and pre-sheath structures established in a flowing plasma, it is necessary to simulate a very large region around the probe. With that objective in mind, we divide the space around the object into three regions, as seen on Fig. 1. Regions A and B, defined by radiuses $R_{A}$ and $R_{B}$ respectively, are part of the computational domain, while region $\mathrm{C}$ contains the background plasma. Following are descriptions of the assumptions made in each of the regions:

Region A This innermost region extends from $r=0$ to $r=R_{A}$ and is the smallest region enclosing the probe, the sheath and part of the pre-sheath. In this region, charge densities are computed through a full kinetic treatment of Vlasov's equation, assuming that the plasma is collisionless. In other words, binary short-range collisions among particles are deemed insignificant as compared to the large electric field forces resulting from the collective longrange Coulomb interactions. Vlasov's equation is solved by tracking the electron and ion trajectories back in time, all the way to the outer boundary at $r=R_{B}$ through regions $\mathrm{A}$ and $\mathrm{B}$. No assumptions are made with regards to the potential structure in region $\mathrm{A}$, but an outer boundary condition is specified by the assumed potential profile in region $\mathrm{B}$.

Region $\mathrm{B}$ This intermediate region extends from $r=R_{A}$ to $r=R_{B}$ and covers the bulk of the presheath. In this region, the electric field is still strong 
enough to overwhelm the effect of binary collision forces. Thus, the particle orbits followed from region A into region B still obey collisionless trajectories. In this region, however, the electric potential follows an assumed shape:

$$
\Phi(r, \theta)=\frac{\Phi_{M}(\theta) R_{A}}{r}
$$

where $\Phi_{M}(\theta)$ is the unknown potential profile on the interface between region $\mathrm{A}$ and region $\mathrm{B}$ at $r=R_{A}$. This assumed profile follows from the asymptotic result obtained for a round cylinder immersed in a non-flowing plasma (derived by Bernstein ${ }^{5}$ and used by Laframboise $\left.{ }^{6}\right)$. It assumes that the variables $r$ and $\theta$ are separable for sufficiently large values of $R_{A}$. Any rotational asymmetries found in the potential profile at the edge of region $\mathrm{A}\left(r=R_{A}\right)$ are propagated into region $\mathrm{B}$, neglecting the damping of the $\theta$-variation of the potential in region $B$. The latter approximation could eventually be improved, but a convergence analysis of our results as a function of the size of region $\mathrm{A}$ will be performed to insure that this approximate behavior does not reflect in our converged results. An improvement in this approximation would merely help reducing the size required on region A for proper convergence.

Region C This region contains the background plasma. It extends beyond the computational domain, from $r=R_{B}$ to $r=\infty$. In this region, electric fields are assumed to have dropped to a low level, such that collision forces are dominating electron behavior. Due to the dominant collisions beyond $r=R_{B}$ and the small value of the electric potential at the outer boundary $r=R_{B}$, we can safely assume a drifting Maxwell-Boltzmann velocity distribution for the electrons at this location:

$$
\begin{aligned}
& f_{e}\left(v_{x}, v_{y}\right)=\frac{n_{0} m_{e}}{2 \pi e T_{e}} \times \\
& \exp \left\{\frac{\Phi_{o b}}{T_{e}}-\frac{m_{e}}{2 e T_{e}}\left(\left(v_{x}-v_{f}\right)^{2}+v_{y}^{2}\right)\right\}
\end{aligned}
$$

where $n_{0}$ is the background plasma density and $v_{f}$ is the speed of the flow, which is negligible for electrons in the mesosonic regime.

The electric field level at which collisional forces are on par with field forces can be defined as the following: an electric field that generates sufficient accelerations to significantly affect the thermal equilibrium over the same time interval as the mean time between collisions, that is, the relaxation time over which velocity distributions are thermalized to a Maxwellian equilibrium. Equating the mean time between collisions with the "equilibrium disturbance" time interval, which is roughly equal to the time required to accelerate a still electron to the average $2 \mathrm{D}$ velocity, we obtain:

$$
\begin{aligned}
\tau & \approx \frac{\bar{v}}{e E / m_{e}} \\
\frac{\lambda_{m f p}}{\bar{v}} & \approx \frac{\bar{v}}{e E / m_{e}} \\
E & \approx \frac{m_{e} \bar{v}^{2}}{e \lambda_{m f p}}=\frac{\pi}{2} \frac{m_{e} v_{t h}^{2}}{e \lambda_{m f p}} \\
E & \approx \frac{\pi}{2} \frac{T_{e}}{\lambda_{m f p}}
\end{aligned}
$$

where we have used the $2 \mathrm{D}$ average velocity $\bar{v}=$ $\sqrt{\frac{\pi}{2}} v_{t h}$, since the $z$ component of the electric field is assumed to be negligible. The mean free path of the dominating collision mechanism having a thermalizing effect on electrons is used here, e.g. the mean free path for electron-electron or electron-neutral collisions (or any other electron scattering mechanism).

As for the ions, their trajectories are assumed collisionless because of their high speed as compared to their temperature, which translates into dominating inertial forces. Ions are assumed to be distributed according to a drifting Maxwellian distribution at infinity, and to follow straight-line collisionless trajectories within region $\mathrm{C}$. In other words, they are accelerated in the direction of their initial velocity at $r=\infty$ until they reach $r=R_{B}$, after which they follow collisionless trajectories guided by the potential distribution in regions $\mathrm{B}$ and $\mathrm{A}$. We assume for their velocity distribution at infinity $(r=\infty)$ :

$$
\begin{aligned}
f_{i}\left(v_{x}, v_{y}\right)= & \frac{n_{0} m_{i}}{2 \pi e T_{i}} \times \\
& \exp \left\{-\frac{m_{i}}{2 e T_{i}}\left(\left(v_{x}-v_{f}\right)^{2}+v_{y}^{2}\right)\right\}
\end{aligned}
$$

Finally, the electric potential is assumed to vary piecewise-bilinearly over the whole computational domain, i.e. it varies bilinearly within any of the mesh's triangles. This simplifies the analytical tracking of sub-trajectories, since the electric field is consequently piecewise-constant. Sufficient meshing accuracy will ensure an accurate representation of the fields.

\section{Formulation of the Poisson-Vlasov Operator}

Obtaining a simultaneous solution in two dimensions for the asymmetric steady-state electric potential distribution and charge density distributions of both plasma species (ions and electrons) requires the ability to solve, self-consistently, Vlasov's equation for each species and Poisson's equation for the 
electric potential and charges, while satisfying appropriate boundary conditions.

Both the electric potential and charge densities are sampled on a piecewise-triangular mesh, which is generated using the Bidimensional Anisotropic Mesh Generator $(B A M G)^{16}$ developed at INRIA, France. An adaptive process was implemented whereby the $B A M G$ mesher is used to periodically refine the mesh based on the approximate net charge density and potential distributions.

At the nodes of this mesh are collocated samples of the continuous ion density distribution $n_{i}(x, y)$, electron density distribution $n_{e}(x, y)$, net charge density distribution $\rho(x, y)=e\left(n_{i}(x, y)-n_{e}(x, y)\right)$ and electric potential distribution $V(x, y)$. These samples are packed into the vectors $\vec{n}_{i}, \vec{n}_{e}, \vec{\rho}$ and $\vec{V}$, respectively. In the remainder of this paper, these sample vectors will be loosely referred to as "distributions" of the corresponding physical quantities.

A Poisson solver $\vec{f}_{P}(\cdot)$ is derived, based on Poisson's equation and the boundary conditions, that solves for the electric potential distribution for a given net charge density distribution $\vec{\rho}$, i.e.:

$$
\vec{V}=\vec{f}_{P}(\vec{\rho})
$$

Likewise, a Vlasov solver $\vec{f}_{V}(\cdot)$ is derived based on Vlasov's equation and the boundary conditions, that solves for the density distributions of both species, for a given electric potential distribution:

$$
\vec{\rho}=\vec{f}_{V}(\vec{V})
$$

Given the Poisson and Vlasov operators, the whole problem can be specified either as a function of the net charge density distribution or the potential distribution. Here we choose to work in terms of the net charge density distribution $\vec{\rho}$, due to a stability advantage inherent to the regularization process discussed later. When combined together, both solvers form a Poisson-Vlasov operator. The solution lies at the fixed point of this operator, i.e. is the root of the following equation:

$$
\vec{\rho}=\vec{f}_{P V}(\vec{\rho}), \text { with } \vec{f}_{P V}(\cdot)=\vec{f}_{V}\left(\vec{f}_{P}(\cdot)\right)
$$

where $\vec{f}_{V}, \vec{f}_{P}$ and $\vec{f}_{P V}$ are the Vlasov, Poisson and $\mathrm{PV}$ operators. In order to get to the solution, we must thus find the root of (12), a nonlinear vector equation.

\section{Poisson solver based on the FEM}

The Poisson solver, which computes a potential distribution from a given net charge density distribution, was implemented using a formulation based on the well-known Finite-Element Method as applied to Poisson's equation. The Poisson solver looks for a solution for the potential profile $V(x, y)$ which satisfies:

$$
\begin{aligned}
\epsilon_{0} \nabla^{2} V(x, y) & =\rho(x, y) \\
\left.V\right|_{\text {conductive surface }} & =V_{0} \\
\left.\frac{\partial V}{\partial r}\right|_{R=R_{A}, \theta} & =-\frac{V\left(R_{A}, \theta\right)}{R_{A}}
\end{aligned}
$$

where the last 2 equations are boundary conditions at the probe and at the outer boundary of region $\mathrm{B}$, respectively.

The details of the derivation are not shown here since this is a standard Finite Element implementation of Poisson's equation subject to mixed boundary condition. ${ }^{17}$ We have used piecewise bilinear charge density and potential distributions in the formulation. This approach has proved more robust and flexible (in terms of applying mixed boundary conditions) than the point-matching technique used previously. ${ }^{18}$

\section{Vlasov solver}

The Vlasov solver described here allows each species to express the full kinetic nature of its $2 \mathrm{D}$ velocity distribution. This feature allows for the realistic and accurate modeling of non-thermal plasmas, that is, plasmas that are not at thermal equilibrium due to large electric fields and high velocity flows. It accounts for the largest part of the computational complexity of the technique presented in this paper.

Assuming a collisionless, quiescent, unmagnetized plasma in a steady state, Vlasov's equation states that the velocity distribution function $f_{e, i}\left(x, y, v_{x}, v_{y}\right)$ for any given plasma species is constant along particle orbits in a given electric potential distribution: ${ }^{19}$

$$
\begin{array}{r}
\frac{d f_{e, i}}{d t}=v_{x} \frac{\partial f_{e, i}}{\partial x}+v_{y} \frac{\partial f_{e, i}}{\partial y}+\frac{q_{e, i}}{m_{e, i}} \frac{\partial V}{\partial x} \frac{\partial f_{e, i}}{\partial v_{x}} \\
+\frac{q_{e, i}}{m_{e, i}} \frac{\partial V}{\partial y} \frac{\partial f_{e, i}}{\partial v_{y}}=0
\end{array}
$$

where we substituted the electric field components with the gradient of the electric potential, i.e. $\vec{E}=$ $-\nabla V$. The indices $e$ and $i$ refer to electrons and ions, respectively. $f_{e, i}\left(x, y, v_{x}, v_{y}\right)$ is the velocity distribution of a given species and $V(x, y)$ is the electric potential distribution function. Given a known potential distribution $V(x, y)$ and a boundary condition for the incoming particles on the outer boundary, the method of characteristics can be used to solve (16) for the velocity distributions $f_{e, i}\left(x, y, v_{x}, v_{y}\right)$ of both the electrons and ions. The 


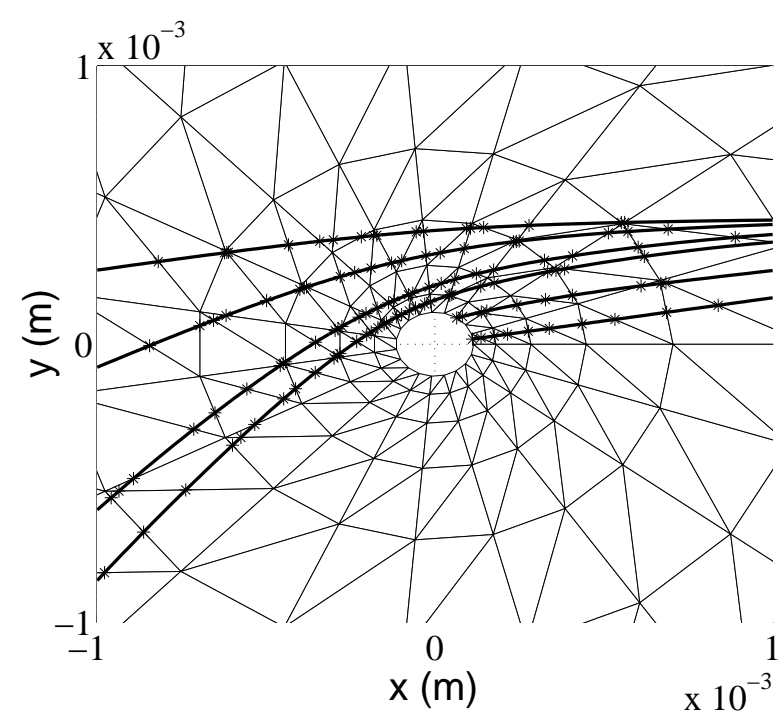

Fig. 2 Example of the Semi-analytical Particle Tracking Process Through the Potential Mesh.

densities of both species are then obtained by integrating over all velocity space:

$$
n_{e, i}=\iint f_{e, i}\left(x, y, v_{x}, v_{y}\right) d v_{x} d v_{y}
$$

and the total charge density results from $\rho(x, y)=$ $q_{i} n_{i}-q_{e} n_{e}$, where $q_{i}$ and $q_{e}$ are the ion and electron particle charge.

The use of the method of characteristics for the resolution of Vlasov's equation in plasma problems was reported early on, ${ }^{13}$ and was referred to as the inside-out procedure. It consists of tracking a particle's trajectory back in time until it intersects the outer boundary of computational space (interface between regions $\mathrm{B}$ and $\mathrm{C}$ on Fig. 1), where the velocity distribution function is sampled. Any trajectory not originating from the outer shell is deemed unpopulated. ${ }^{6}$ Such is the case for trajectories originating from the tether itself, which we assume does not emit charged particles, as well as trapped trajectories which have no sources in the collisionless case. However, it should be pointed out that the Maxwell-Boltzmann velocity distribution used for electrons (see Eq. (4)) allows nonetheless some electrons having a total energy less than zero into the computational space; these particle may be regarded as "trapped particles" as well.

Fig. 2 illustrates the particle tracking process. The trajectories are tracked analytically from one edge of the mesh to another, assuming a constant electric field within any given triangle of the mesh. Every sub-trajectory is thus resolved by computing the intersection of a quadratic parametric curve with a segment on the mesh. This technique is much more efficient than using a fixed time step particle pusher, since the amount of computations necessary for one trajectory depends on the number of edges being crossed rather than the number of time steps necessary to reach a boundary. Also, given the assumption of a piecewise-bilinear potential distribution, it provides exact (nearly exact if we account for roundoff errors) conservation of energy along orbits, which contributes to the accuracy of the overall approach.

To obtain a value for the particle density at a given point, one needs to integrate the values obtained for $f_{e, i}\left(x, y, v_{x} e, v_{y}\right)$ using Eq. (17). To limit the computational task, the domain of integration is restricted to a limited region outside of which the velocity distribution function is known to be very low. Conservation of energy, together with knowledge about the velocity distribution functions at the outer boundary, is used to define a annular domain of integration in two-dimensional velocity space, ${ }^{18}$ which corresponds to a rectangular domain of integration in the two-dimensional space defined by the kinetic energy and the angular direction. The integration is performed in the latter $2 \mathrm{D}$ space, using an adaptive cubature of triangular elements. A modified version of routine TOMS706 $6^{20,21}$ from the Transactions on Mathematical Software repository was used to this effect. The main integration subroutine for computing the integral over a single triangular region was substituted with Algorithm 007 from the Journal of Computational Applied Mathematics, using a 64-point generalized Gauss-Legendre product rule. 64 points are thus used to evaluate the integral over each of the triangular elements of the domain in this adaptive triangulation of the kinetic-energy/direction-angle space. Typical calculations involve from 50 to a few hundred triangular elements, therefore requiring several thousand evaluations of the velocity distribution function for each sample of the number density.

In order to obtain an estimate of the error on the number density calculation, the integral over each triangular element is actually computed twice, once using the 64-point rule discussed above, and once using a 49-point rule. The error estimate is taken as the difference between the 2 evaluations. In addition to providing an estimate for the error on the density, these error estimates are used in the adaptive refinement of the triangulation. The error estimate on the density is used as part of the regularization of the Newton iteration, as shown in the following section. 


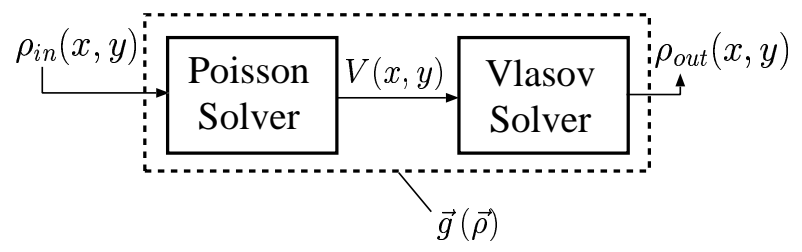

Fig. 3 Fixed Point Operator Comprised of Both the Poisson and Vlasov Solvers.

Tikhonov-regularized Newton Iterative Method

Using the Poisson and Vlasov solvers, we seek a solution for the potential and charge distributions that simultaneously satisfies the Poisson and Vlasov equations. Fig. 3 depicts the general fixed-point operator comprised of both the Poisson and Vlasov solvers. The fixed-point operator takes a charge distribution $\vec{\rho}_{i n}$ at its input and generates a new estimate $\vec{\rho}_{\text {out }}$ :

$$
\vec{\rho}_{\text {out }}=\overrightarrow{\mathbf{g}}\left(\vec{\rho}_{\text {in }}\right)
$$

There are known difficulties arising in solving such a problem. ${ }^{6}$ Simple iteration of the fixed point operator does not in general yield convergence, since it is a non-contractive mapping. ${ }^{22}$ Instead, we use a procedure based on Newton's method for nonlinear systems of equations ${ }^{23}$ which is depicted in Fig. 4. This necessitates the Jacobian matrix $\mathbf{J}_{\mathbf{g}}$ of the fixed point operator and is equivalent to iterating the following fixed point function:

$\vec{\rho}_{\text {out }}=\vec{h}\left(\vec{\rho}_{\text {in }}\right)=\vec{\rho}_{\text {in }}+\left(\mathbf{J}_{\mathbf{g}}\left(\vec{\rho}_{\text {in }}\right)-\mathbf{I}\right)^{\dagger}\left(\vec{\rho}_{\text {in }}-\overrightarrow{\mathbf{g}}_{\left.\left(\vec{\rho}_{\text {in }}\right)\right)}\right.$

where the symbol $\dagger$ represents a regularized inversion based on column normalization and Tikhonov regularization, ${ }^{24}$ both of which are necessary to filter out the errors introduced by the Vlasov solver. These errors originate from the finite precision with which densities are computed, combined with the fact that like numbers are subtracted to obtain the net charge density in the pre-sheath where the ion and electron densities are very close to each other.

$\mathbf{J}_{\mathbf{g}}\left(\vec{\rho}_{i n}\right)$ is the Jacobian matrix of operator $\mathbf{g}(\cdot)$ evaluated at $\vec{\rho}_{i n}$. If there are $N$ unknowns ( $N$ charge nodes), the Jacobian matrix is a $N \times N$ matrix and is defined by:

$$
\mathbf{J}_{\mathbf{g}}(i, j)=\frac{\partial g_{i}}{\partial \rho_{j}}, \quad i=1, \ldots, N, \quad j=1, \ldots, N
$$

Using finite differences to compute an approximation for $\mathbf{J}_{\mathbf{g}}$ would be prohibitively expensive computationally. Instead, direct computation of $\mathbf{J}_{\mathbf{g}}$ is performed. This involves combining the gradients of every sample of the velocity distribution function that were obtained at that particular iteration, and

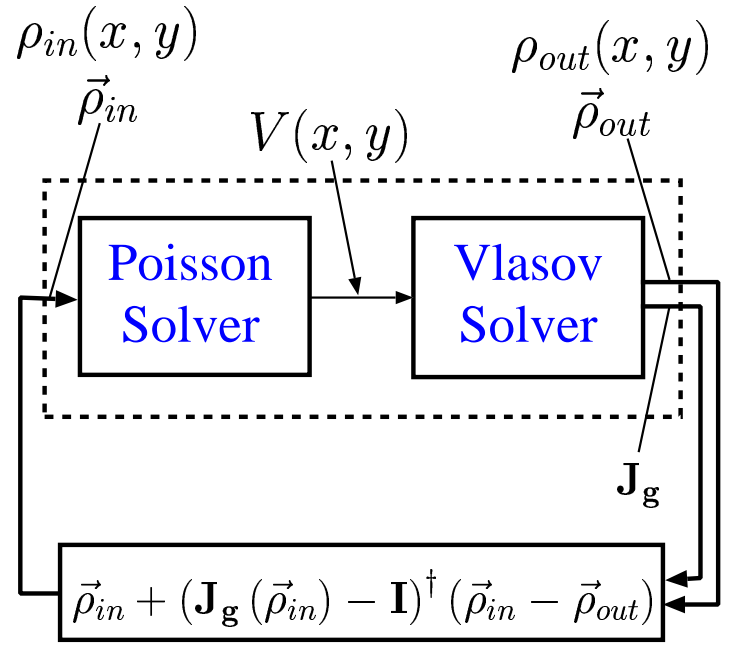

Fig. 4 Tikhonov-regularized Newton Iterative Poisson/Vlasov Procedure

replicating all the operations that were applied to those samples. To obtain the gradients of each velocity distribution sample, the analytical gradient of the velocity distribution at the outer boundary is propagated and transformed along the corresponding particle trajectory. To reduce the computation time of each iteration, the Jacobian computed at the first iteration may be used for subsequent iterations, a technique know as the Chord method. ${ }^{25}$ This has the disadvantage of increasing the number of required iterations, but it stabilizes the Newton iterations by avoiding the noise generated due to the finite precision of each Jacobian computation.

The principal difficulty involved in the regularization process is the choice of the regularization parameter. Both the regularization parameter and the precision of the Vlasov solver must be selected such that the "Vlasov noise" will penetrate the solution at a slower rate than the rate of convergence of the process. Details of the implementation of this regularization procedure will be presented in an upcoming journal article.

\section{Parallel Implementation}

The iterative resolution high-level algorithm and the Poisson solver, both fairly light computationally, were implemented in Matlab. The Vlasov solver, being much heavier computationally, was implemented in Fortran 90 using an MPMD* parallel processing scheme based on the Parallel Virtual Machine library(www.epm.ornl.gov/pvm/pvm_home.html). The program is being run on two different platforms. The primary platform is the AMD Linux

\footnotetext{
* Multiple Program, Multiple Data
} 
cluster at the University of Michigan's Center for Advanced Computing, accessed through the National Partnership for Advanced Computational Infrastructure (NPACI). The secondary platform is based on opportunistic pooling of local Linux and Sun workstations. The parallelization is implemented at a fairly high level: each node is assigned the task of computing one or more samples of the number density. Twenty processors are used in a typical simulation on the Linux cluster.

\section{Simulation Results}

Earlier validation runs ${ }^{18}$ had confirmed that our code has excellent agreement with OML theory for both electron and ion collection in a non-flowing plasma, in saturation as well as in retardation regimes.

Here we present recent results obtained for a long probe with a one-Debye-length radius, immersed in a Xenon plasma. The flow energy is 14 times the electron temperature, and the applied bias is 40 times the electron temperature. For this simulation, region A had a radius of 100 Debye lengths, and region $\mathrm{B}$ extended to a radius of 1514 Debye lengths. Based on Eq. (8), the electric field at the outer edge of region $B$ has a magnitude such that the forces it creates on the electrons are equivalent to that caused by a collisional process having a mean free path between 21000 and 26000 Debye lengths. The extent of region B was actually sized with the intent of achieving an edge electric field equivalent to a 19000 Debye length mean free path, which is the theoretical value for the electron-neutral collisions corresponding to the background pressure and plasma parameters of a vacuum experiment reported in Ref. 26.

Fig. 5 shows two different views of the various profiles obtained at the $200^{\text {th }}$ iteration of the simulation. The plasma flows along the $x$ axis (shown here in scaled form as the $u$ axis) from negative to positive $x$ 's. The charge distribution $\rho_{i n}$ and potential distribution correspond to the input and output of the Poisson solver, respectively. The "ion density" and "electron density" profiles correspond to the output of the Vlasov solver, and are combined to produce the net charge output $\rho_{\text {out }}$ of the Poisson-Vlasov operator.

The convergence parameter (shown in Fig. 5) is expressed in the form of a relative difference between the input and output net charge density distributions. Here the error was reduced down to $22.5 \%$. As discussed earlier, the electron and ion density profiles generated by the Vlasov solver are computed with finite precision. Despite the regularization, some of the noise present in the net charge $\rho_{\text {out }}$ gets inserted into the solution $\rho_{i n}$, and builds up over the iterations. The present simulation was not iterated any further than 200 iterations, in order to limit the amount of noise that gets inserted into the solution. Further work is needed to reduce the amount of noise generated by the quadrature integration process in the Vlasov solver and allow us to reduce the error below $1 \%$.

The results indicate an enhancement in the collected current as compared to the OML-predicted level of current $\left(I=7.76 I_{\text {the }}\right.$ as opposed to $I_{o m l}=$ $\left.7.23 I_{\text {the }}\right)$. Various numerical experiments have indicated that there are two opposing flow-related phenomena that can be associated with the change in the amount of collected current as compared to OML theory:

1. the potential depression seen on the wake side of the probe acts as a potential barrier that prevents some electrons from reaching the probe, thereby reducing the amount of collected electron current. The very low electron number density seen on the wake side provides confirmation of that phenomenon;

2. upstream from the probe, the electric potential has a small but finite value on the edge of computational space (in the case presented here, the upstream edge potential is $0.11 T_{e}$ ). Still, the electric field at this location is sufficiently weak that collisions dominate the motion of electrons, based on a mean free path as large as 21000 to 26000 Debye lengths as mentioned above. The density of the electrons incoming from the collisional region $\mathrm{C}$ (see Fig. 1) is given by a Maxwell-Boltzmann law (see Eq. 4), owing to the collisional nature of the electrons beyond our computational space. Thus, the density of the incoming electrons from the ram side is proportional to $e^{\frac{\phi_{e d g e}}{T_{e}}} \approx e^{0.11}=1.12$, which increases the amount of collected electron current. This phenomenon also allows the electron density to rise above the background plasma density, as seen on Fig. 5. The plasma flow creates an elongation of the pre-sheath in the direction of the flow, which causes, on the ram side, the electric field to drop below the "collisional influence" limit before the potential as reached a small value, thereby causing an enhancement in the number density of incoming electrons that are transitioning from collisional region $\mathrm{C}$ to collisionless region $\mathrm{B}$.

It appears that, in the present case, the second ef-

8 OF 11

American Institute of Aeronautics and Astronautics 

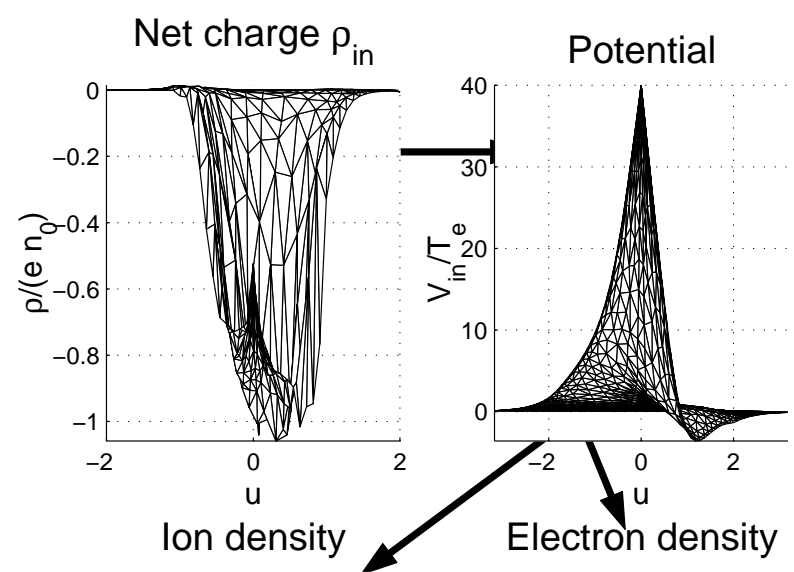

iteration \#200/200
$X-Y$ AXES LOG SCALE:

$$
\begin{aligned}
& u=\log _{10}\left(r / r_{0}\right) x / r \\
& v=\log _{10}\left(r / r_{0}\right) y / r
\end{aligned}
$$

$\left\|\rho_{\text {out }}-\rho_{\text {in }}\right\| /\left\|\rho_{\text {in }}\right\|=0.225$

Normalized Collected Current:

$$
\mathrm{I} / \mathrm{I}_{\text {the }}=7.761>\mathrm{I}_{\text {oml }} / \mathrm{I}_{\text {the }}=7.225
$$

Net charge $\rho_{\text {out }}$

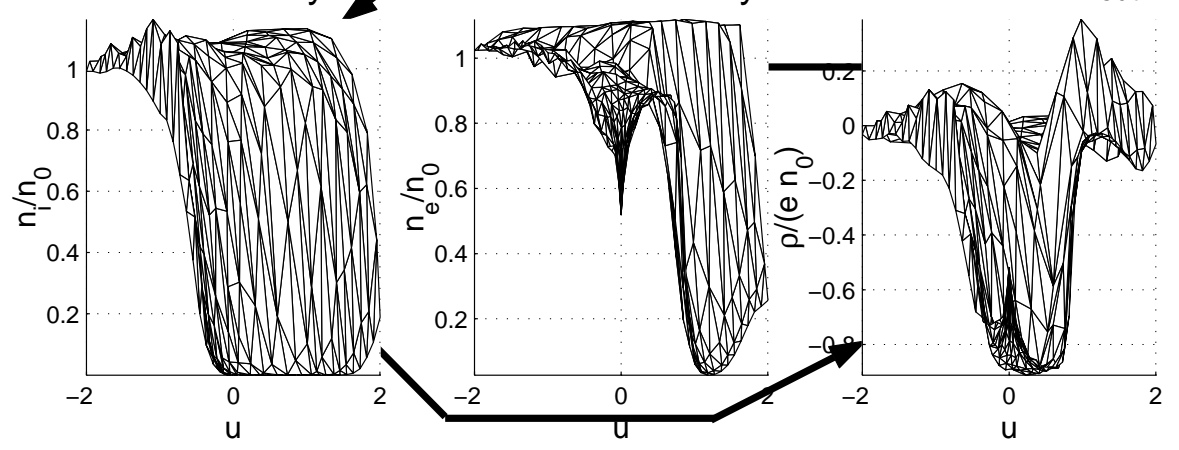

(a)

\section{Net charge $\rho_{\text {in }} \quad$ Potential iteration \#200/200}

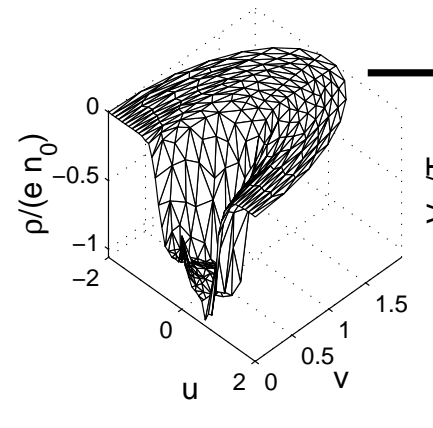

Ion density

$$
\begin{gathered}
\text { X-Y AXES LOG SCALE: } \\
u=\log _{10}\left(r / r_{0}\right) x / r \\
v=\log _{10}\left(r / r_{0}\right) y / r
\end{gathered}
$$

$$
\left\|\rho_{\text {out }}-\rho_{\text {in }}\right\| /\left\|\rho_{\text {in }}\right\|=0.225
$$

Normalized Collected Current:

$$
\mathrm{I} / \mathrm{I}_{\text {the }}=7.761>\mathrm{I}_{\text {oml }} / \mathrm{I}_{\text {the }}=7.225
$$

Net charge $\rho_{\text {out }}$

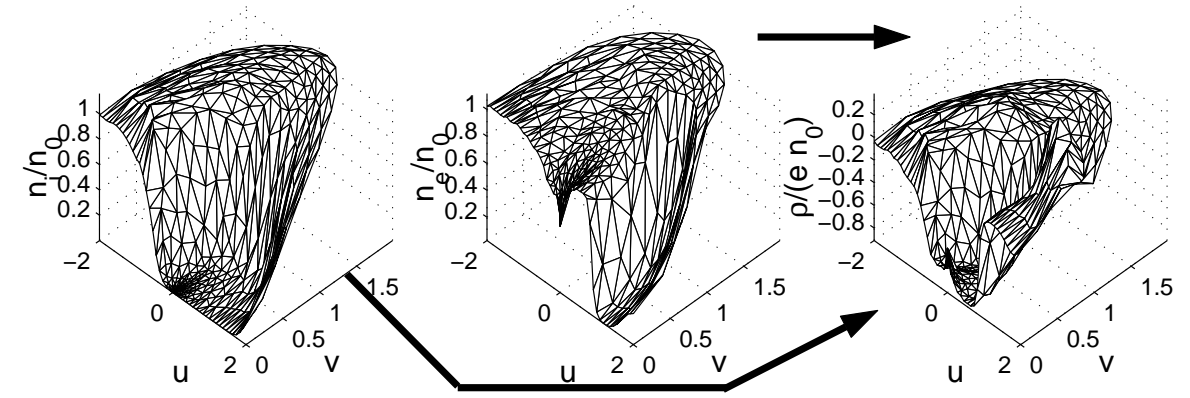

(b)

Fig. 5 Simulation result shown for a 1-Debye-length radius probe biased at $40 T_{e}$ and immersed in a Xenon flowing plasma with beam energy of $14 T_{e}$. To help the visualization of the results, the $x$ and $y$ axes were "stretched" based on the following mappings: $u=\log _{10}\left(r / r_{0}\right) x / r$ and $v=\log _{10}\left(r / r_{0}\right) y / r$; (a) side view (b) slanted view. 
fect dominates the first one, resulting in an overall current enhancement. This observed enhancement is qualitatively consistent with recent experimental results, although not as important $(7.4 \%$ in these simulation results as opposed to $39 \%$ in our experimental results ${ }^{26}$ ). This difference in the magnitude of the enhancement might be explained by a shorter thermalizing collision mean-free-path in our experiment as compared to the one used in the simulation to determine the extent of region $B$. One of the reasons for this discrepancy could be that the background neutral pressure within the plasma beam of these experiments was actually higher than the measured value used to determine the theoretical mean free path of 19000 Debye lengths that was referred to above. The background pressure was measured at another location within the $6 \mathrm{~m} \times 9 \mathrm{~m}$ vacuum chamber, out of the plasma beam. Clearly, a smaller mean-free-path would increase the collected current in our simulation.

In a previous communication, ${ }^{18}$ we had shown simulation results indicating a collection current below OML. However, the model used then assumed the presence of a localized ground at some distance from the tether and did not allow for the electron density enhancements consistent with a slow increase of the potential upstream from the probe combined with thermalizing collisions. Out of the two phenomena identified above, only the first one (i.e., a potential depression on the wake side) was then simulated, thus resulting in a reduced collected current due to potential barriers.

\section{Present Status and Conclusions}

We have presented a steady-state 2 -species plasma model applicable to arbitrary 2D conductive objects in flowing plasmas with very long electron collision mean free paths and collisionless ions. The effects of collisions were addressed by dividing the space surrounding the conductive object (the probe) into a collisionless computational space (regions A and B) and a collisional background plasma (region $\mathrm{C}$ ).

Results were shown pertaining to the case of a circular cylinder in a flowing plasma, with indication of a small enhancement of the collected current with respect to OML theory. This enhancement is attributed to the elongation of the pre-sheath into the collisional zone of the plasma, which causes an enhanced density of incoming electrons upstream from the probe, and is seen to dominate the opposing decrease in electron collection due to additional potential barriers created by a wake-side depression of the electric potential.

The primary issue that will need to be addressed is the accumulation of noise in the solution that subsides in spite of the employed Tikhonov regularization. Improvements must be made in the Vlasov solver in order to reduce the amount of quadrature noise generated and consequently reduce the error level, thereby improving the consistency of the solution. Further developments will also address noncircular cross-sections.

\section{Acknowledgments}

The authors would like to thank Dr. Dave Cooke, Dr. Ira Katz and Dr. Juan Sanmartín for useful discussions on the topic. É.C. acknowledges the scholarship support of the Horace H. Rackham School of Graduate Studies at the University of Michigan, the Natural Sciences and Engineering Research Council of Canada and the Communications Research Centre(Canada).

\section{References}

${ }^{1}$ Estes, R., Lorenzini, E., Sanmartín, J., Peláez, J., Martínez-Sánchez, M., Johnson, C., and Vas, I., "Bare Tethers for Electrodynamic Spacecraft Propulsion," Journal of Spacecraft and Rockets, Vol. 37, No. 2, March-April 2000, pp. 205-211.

${ }^{2}$ Sanmartín, J., Martínez-Sánchez, M., and Ahedo, E., "Bare Wire Anodes for Electrodynamic Tethers," Journal of Propulsion and Power, Vol. 9, No. 3, 1993, pp. 353-360.

${ }^{3}$ Estes, R. and Sanmartín, J., "Cylindrical Langmuir probes beyond the orbital-motion-limited regime," Physics of Plasmas, Vol. 7, No. 10, October 2000, pp. 4320-4325.

${ }^{4}$ Mott-Smith, H. and Langmuir, I., "The Theory of Collectors in Gaseous Discharges," Physical Review, Vol. 28, 1926, pp. $727-763$.

${ }^{5}$ Bernstein, I. and Rabinovitz, I., "Theory of Electrostatic Probes in a Low-Density Plasma," Physics of Fluids, Vol. 2, No. 2, March-April 1959, pp. ?-?

${ }^{6}$ Laframboise, J., Theory of Sperical and Cylindrical Langmuir Probes in a Collisionless, Maxwellian Plasma at Rest, Ph.D. thesis, University of Toronto, June 1966.

${ }^{7} \mathrm{Kanal}, \mathrm{M}$. , "Theory of current collection of moving cylindrical probes," Journal of Applied Physics, Vol. 35, 1964, pp. 1697.

${ }^{8}$ Hoegy, W. and Wharton, L., "Current to a Moving Cylindrical Electrostatic Probe," Journal of Applied Physics, Vol. 44, No. 12, December 1973, pp. 5365-5371.

${ }^{9}$ Godard, R. and Laframboise, J., "Total Current To Cylindrical Collectors in Collisionless Plasma Flow," Planetary Science, Vol. 31, No. 3, 1983, pp. 275-283.

${ }^{10}$ Langdon, C. B. . A. B., Plasma Physics Via Computer Simulation, IOP Publishing, 1991.

${ }^{11}$ Filbet, F. and Sonnendrücker, E., "Comparison of Eulerian Vlasov Solvers," Comp. Phys. Comm., Vol. 150, No. 3, 2003, pp. 247-266.

${ }^{12}$ Parker, L. and Sullivan, E., "Numerical Solution of the Poisson-Vlasov System for a Spherical Electrostatic Probe," SIAM Fall Meeting, October 1970.

${ }^{13}$ Parker, L. W., "Computation of Collisionless SteadyState Plasma Flow Past a Charged Disk," Tech. rep., Lee W. Parker, Inc., 1976, Prepared for NASA - George C. Marshall Space Flight Center.

10 of 11 
${ }^{14}$ Tautz, M., Cooke, D., Rubin, A., and Yates, G., "Preliminary Documentation of the MACH Code," Tech. rep., Hanscom Air Force Base, Septembre 1987.

${ }^{15}$ Geist, A., Beguelin, A., Dongarra, J., Jiang, W., Manchek, R., and Sunderam, V., PVM 3 User's Guide and Reference Manual, Oak Ridge National Laboratory, September 1994 .

${ }^{16}$ Hecht, F., "Bidimensional Anisotropic Mesh Generator," Tech. rep., INRIA (France), 1998.

${ }^{17}$ Tong, P. and Rossettos, J. N., Finite-Element Method Basic Technique and Implementation, chap. 2: The FiniteElement Method for Poisson's Equation, The MIT Press, 1977, pp. 40-71.

${ }^{18}$ Choinière, E. and Gilchrist, B. E., "Electron Collection to Arbitrarily Shaped Electrodynamic Tethers in Flowing Plasmas: a Kinetic Model - Validation for Circular Crosssections," AIAA Paper AIAA-2002-4050, July 2002.

${ }^{19}$ Lieberman, M. A. and Lichtenberg, A. J., Principles of Plasma Discharges and Materials Processing, Wiley Interscience, 1994.

${ }^{20}$ and Terje O. Espelid, "Algorithm 706-DCUTRI: An Algorithm for Adaptive Cubature Over a Collection of Triangles," Transactions on Mathematical Software, Vol. 18, No. 3, September 1992, pp. 329-342.

${ }^{21}$ Espelid, T. O., "Remark on Algorithm 706: DCUTRI An Algorithm for Adaptive Cubature Over a Collection of Triangles," Transactions on Mathematical Software, Vol. 24, No. 3, September 1998, pp. 334-335.

${ }^{22}$ Sikorski, K. A., Optimal Solution of Nonlinear Equations, Oxford University Press, 2001.

${ }^{23}$ Burden, R. L., Numerical Analysis, PWS-KENT Publishing Company, 4th ed., 1989.

${ }^{24}$ Hansen, C., Regularization Tools - A Matlab Package for Analysis and Solution of Discrete Ill-Posed Problems, University of Denmark, 2001.

${ }^{25}$ Rheinboldt, W. C., Methods for Solving Systems of Nonlinear Equations, SIAM, 2nd ed., 1974.

${ }^{26}$ Choinière, E., Gilchrist, B. E., Bilén, S. G., and Fuhrhop, K. R., "Measurement of Cross-Section Geometry Effects on Electron Collection to Long Probes in Mesosonic Flowing Plasmas," AIAA Paper AIAA-2003-4950, 2003. 